\title{
Design, Simulation, and Optimization of an Irregularly Shaped Microstrip Patch Antenna for Air-to-Ground Communications
}

\author{
L. A. R. Ramirez and J. C. A. Santos \\ Electrical Engineering Department, Military Institute of Engineering (IME), Rio de Janeiro, RJ, Brazil \\ Correspondence should be addressed to J. C. A. Santos; araujo@ime.eb.br
}

Received 22 December 2016; Revised 20 February 2017; Accepted 5 March 2017; Published 28 March 2017

Academic Editor: Sotirios K. Goudos

Copyright (c) 2017 L. A. R. Ramirez and J. C. A. Santos. This is an open access article distributed under the Creative Commons Attribution License, which permits unrestricted use, distribution, and reproduction in any medium, provided the original work is properly cited.

\begin{abstract}
In this study an irregularly shaped microstrip patch antenna was designed, simulated, and optimized for air-to-ground communication (ATG) applications. The process started with the design of a rectangular patch antenna with the traditional transmission line and cavity methods, followed by a simulation with the finite-difference time-domain method (FDTD) in conjunction with a genetic algorithm (GA). The aim of the study was to design an efficient patch antenna. The designed antenna is resonating at $14.25 \mathrm{GHz}$ with $35 \mathrm{~dB}$ return loss. The $10 \mathrm{~dB}$ bandwidth of the antenna is $3.7 \mathrm{GHz}$.
\end{abstract}

\section{Introduction}

The use of microstrip structures to radiate electromagnetic waves started in the 1950s [1]. The earliest form of planar antennas, integrated with planar transmission lines, was developed by Deschamps and Sichak [2]. Practical implementation started to increase in the 1970s when suitable substrate materials became available. The development of microstrip antennas in the 1970s and 1980s has been well documented by Bahl and Bhartia [3] and James and Hall [4], and later several good books were published on this subject [5-16].

A microstrip patch antenna in its simplest configuration consists of a radiating patch on one side of a dielectric substrate and a ground plane on the other side. With regard to the shape, microstrip antenna patches can be regular or irregular. Typical regular shapes are rectangular, square, circular, elliptic, and triangular, among others. These shapes have been widely studied and the design procedures are well established. They are used as conformal antennas for airplanes, cars, missiles, portable devices and many other applications. Regularly shaped antennas are more common, mainly because they are easy to analyze and usually present a symmetric radiation pattern. The irregularly shaped patch antenna refers to a microstrip antenna whose patch geometry is designed to fulfill a specific antenna property such as compactness, wideband characteristics, and multiresonant operation. Examples include H-Shape, E-Shape, and L-Shape, as well as combinations of these antennas [3-16]. In the past few years, patch antennas with nonintuitive and novel shapes have appeared. The radiating patch is modified through an optimization process, aimed at improving its specific properties, in particular increasing the bandwidth [17-22].

In [17], Michael Johnson and Rahmat-Samii described the technique of combining GA optimization with method of moments for broadband patch antenna design. Their study included the optimization of a simple patch antenna to produce a wider operational bandwidth than classical designs. Specifically, the goal was to produce an irregularly shaped patch antenna with a voltage standing wave ratio (VSWR) of $2: 1$ over $20 \%$ bandwidth centered at $3 \mathrm{GHz}$. The GA optimized the subpatch by removing the square metal sections from the patch region. Choo et al. [18] designed a broadband patch antenna using an approach similar to that described in [17]. They began with a metal patch, in which subpatches were represented by Boolean subpatches, either ones (metal) or zeros (nonmetal). The goal was to broaden the bandwidth of a microstrip antenna around the center frequency of $2 \mathrm{GHz}$ by changing the patch shape. Chapter 32 of a handbook published by Balanis [19] presents an overview of antenna optimization using GA and provides some cases of use and a selected bibliography. In [20], Griffiths et al. 


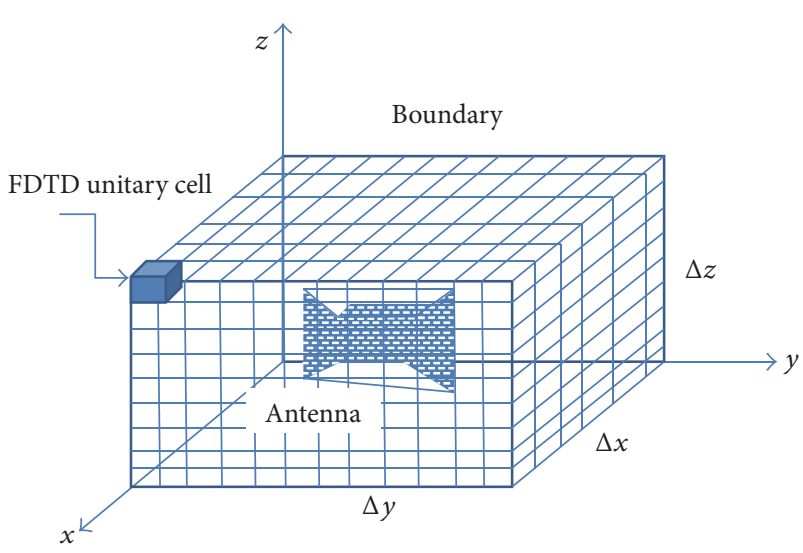

(a) FDTD mesh or computational grid

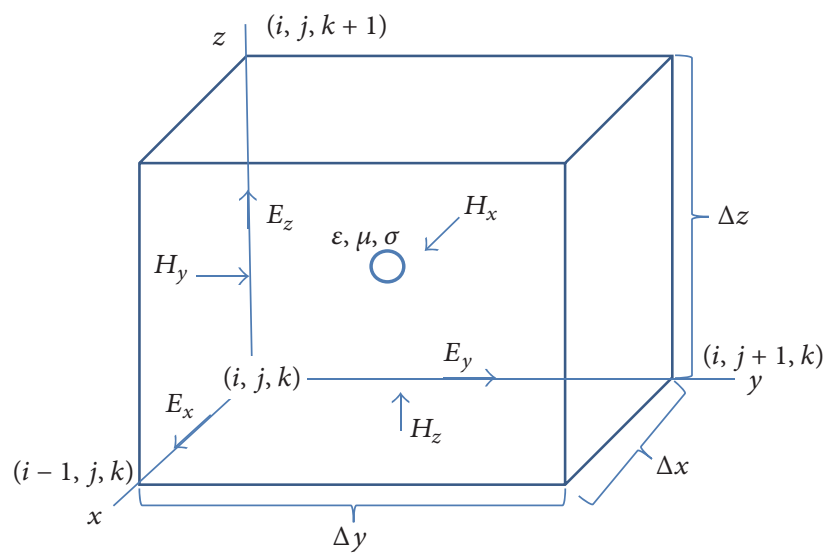

(b) FDTD unitary cell

FIGURE 1: FDTD (a) grid and (b) field components in a unitary cell.

report the use of GA for the generation of compact antennas (broadband and multiband), where the patch is modified using ellipses to create new forms, with higher bandwidth and return loss. In [21], a semiautomatic antenna design method is proposed to design a multiband monopole antenna. In the antenna optimization, particle swarm optimization (PSO) was used, with a new technique of grid patch generation, and good simulation and measurement results were obtained. In [22], Modiri and Kiasaleh used the binary particle swarm optimization (BPSO) algorithm to design several irregularly shaped microstrip antennas, the main focus being the frequency selectivity of a software-defined radio (SDR).

As in [17-22], an irregularly shaped microstrip antenna is the object of this study; however, here it will be applied to air-to-ground (ATG or A2G) communication.

Ground-to-ground radio communication is a common application of mobile wireless networks. ATG communication has received increased attention recently [23]. It is used for communication between aircraft and stations on the ground or on water. Line-of-sight (LOS) conditions are usually established. Internet service providers also use ATG to connect planes to the Internet, which has significant limitations in terms of the speed and latency of the connection (onboard WiFi allows no video streaming and no fast browsing, phone calls are of poor quality, etc.). ATG systems are less expensive than satellite-based systems and, thus, services can be provided to passengers at lower cost [23]. Also, this technology will enable a much larger set of applications due to the lower latency and cost per bit. Interest is enhanced by the fact that several companies (e.g., Alcatel and Nokia) currently provide 4G services using air-to-ground LTE [24]. This technology has also become of interest for unmanned aerial vehicles (UAVs), commonly known as a drones, for military, agriculture, and surveillance applications [25].

In this paper, we report the design of a microstrip patch antenna for the ATG frequency band, starting from the classical rectangle microstrip antenna with an insetfed line to obtain an improved antenna. In this study, the finite-difference time-domain numerical method FDTD-3D is combined with a genetic algorithm (GA) to optimize the bandwidth and return loss of the antenna. The full wave FDTD-3D method is described in Section 2. Section 3 presents the GA. The design of the antenna is described in Section 4, and the numerical results are reported in Section 5. Finally, Section 6 provides the conclusions.

\section{Finite Differences in Time Domain}

In the finite-difference time-domain method, Maxwell equations in a differential form are transformed into discrete finite-difference equations. This results in a fully explicit system of difference equations, whereby chronological values of the electric and magnetic field components are obtained in a leap-frog manner; that is, the electric field is solved at a given instant of time and the magnetic field is solved at the next time instant. The process is cyclically repeated. Spatially, the calculation of the electric and magnetic field components is dependent only on the neighbors in each case.

For the FDTD-3D case, the computational domain is divided into small cells (Figure 1(a)). The components $\vec{E}$ and $\vec{H}$ are distributed in space, with the components $\vec{E}$ positioned on the edges and components $\vec{H}$ distributed at the center of the faces of the cells (Figure 1(b)) [26].

With this field distribution, with two adjacent cubes connected by a vertex, each field component of $\vec{E}$ is surrounded by four components of $\vec{H}$, and similarly each component of $\vec{H}$ is surrounded by four components of $\vec{E}$, which can be seen in detail in Figure 2.

In linear, homogeneous, isotropic, and free source media, Maxwell equations can be written as

$$
\begin{aligned}
& \nabla \times \vec{E}=-\mu \frac{\partial \vec{H}}{\partial t} \\
& \nabla \times \vec{H}=\sigma \vec{E}+\varepsilon \frac{\partial \vec{E}}{\partial t} .
\end{aligned}
$$




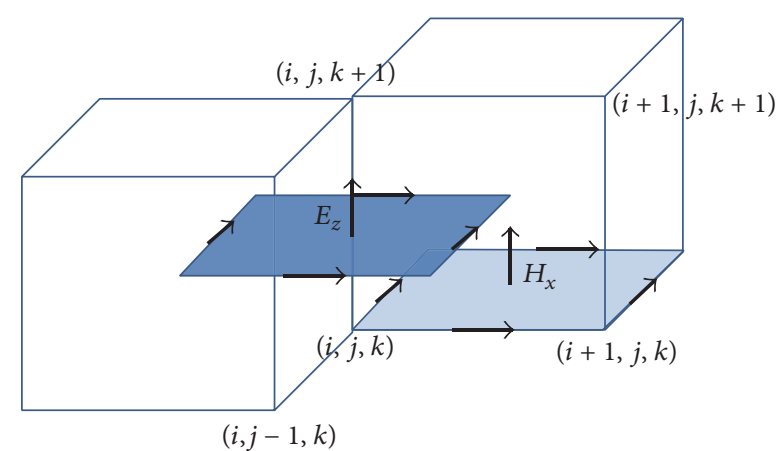

Figure 2: Fields components of $\vec{E}$ and $\vec{H}$ in two adjacent unitary cells.

The spatial and temporal derivatives in (1) are written in a discrete form using a central difference scheme.

The choice of cell size is critical in applying the FDTD method. It must be small enough to permit accurate calculations at the highest frequencies of interest and yet be large enough to ensure that the computational resources are manageable. The cell size is directly affected by the objects in the mesh. Once the cell size is chosen, the maximum time step is determined by satisfying the Courant stability criterion

$$
\Delta t \leq \frac{1}{v_{\max } \sqrt{1 /\left(\Delta x_{\min }\right)^{2}+1 /\left(\Delta y_{\min }\right)^{2}+1 /\left(\Delta z_{\min }\right)^{2}}}
$$

which enforces the property that an incident waveform cannot pass through more than one cell during one time step. This is because during one time step the FDTD algorithm can only propagate across one cell to its nearest neighbor. In (2), $v_{\max }$ is the maximum speed of wave propagation in the model, $\Delta x, \Delta y$, and $\Delta z$ are the space increments, and $\Delta t$ is the time step [26].

Together with the FDTD method, the uniaxial perfectly matched layer (UPML) $[26,27]$ was used. This is an absorbent boundary condition, used to simulate the propagation of waves in free space outside the computational domain, such as an anechoic chamber. Figure 3 illustrates the UPML with an FDTD grid.

\section{Genetic Algorithm}

A genetic algorithm (GA) is an optimization technique that can handle the common characteristics of electromagnetic fields. It is an iterative optimization procedure and it maintains a population of probable solutions (chromosomes) within a search space (which is usually discrete) over many simulated generations.

The basic principles of GA were inspired by the mechanism of natural selection of Darwin and genetic laws, where stronger individuals are likely to be winners (the fittest) in a competing environment and present better characteristics for adaptation [28]. GA assumes that the potential solution of any problem is an individual from a population. Any chromosome can be represented by a set of parameters. These parameters are regarded as the genes and can be structured by a string of values (alleles) in binary form or real values. In a computer algorithm, genes represent the basic building blocks of a genetic algorithm. Figure 4 shows a sample of a binary chromosome and three of its components.

A chromosome (or individual) is an array of genes, and a group of chromosomes is known as a population (see the following).

Matrix representation of components of a genetic algorithm, adapted from [28], is as follows:

$$
\text { population }=\underbrace{\left[\begin{array}{c}
\operatorname{chrom}_{1} \\
\operatorname{chrom}_{2} \\
\operatorname{chrom}_{3} \\
\text { chrom }_{4}
\end{array}\right]}_{\text {chromosomes }}=\underbrace{\left[\begin{array}{cccc}
g_{11} & g_{12} & & g_{1 M} \\
g_{21} & g_{22} & & \\
\vdots & \ddots & \vdots \\
g_{N 1} & \cdots & g_{N M}
\end{array}\right]}_{\text {genes }} \text {. }
$$

Each gene of a chromosome will represent a property of the patch antenna and all genes belonging to a chromosome will represent an antenna configuration. At each iteration (or generation) three basic genetic operations ("selection," "crossover," and "mutation") are performed. The initial population is a randomly generated chromosome satisfying boundary or system constraints to the known configuration that needs to be optimized. A good choice of the population size optimizes the convergence of the simulation.

During each generation, the chromosomes are evaluated, using an associated cost function, and this gives some measurements for the fitness or relative merit. A new generation is formed by selection, according to the fitness values, to create the next generation of individuals from the previous population. These individuals are called parents while those created by applying evolutionary operators to the parents are referred to as offspring. These new chromosomes (offspring) can be formed using several techniques, for instance, by either merging two chromosomes from the current generation using a crossover operator or modifying a chromosome using a mutation operator. In this process, some of the parents and offspring are chosen while others are rejected, so as to keep the population size constant. Fitter chromosomes have a higher probability of being selected. After several generations, the algorithms converge to the best chromosome, which is expected to represent the optimum or suboptimal solution to the problem. Figure 5 shows a flowchart of the GA used.

\section{Microstrip Antenna Design}

4.1. Antenna Design. The first step in the design of the proposed antenna was the dimensioning of a classical rectangular patch antenna through the transmission line and resonant cavity methods [29]. The antenna is shown in Figure 6. The frequency of resonance was chosen in accordance with airto-ground technology. Input data were center frequency $(f 0)$ at $14.25 \mathrm{GHz}$ and frequency band range of 13.25 to $15.25 \mathrm{GHz}$. The three metal components (ground plane, antenna patch, and feed line) have a conductivity of $5.8 \times 107 \mathrm{~S} / \mathrm{m}$ (for copper clad substrates). The substrate RT/duroid 5880 was used, which has a relative permittivity of 2.20. Additional 


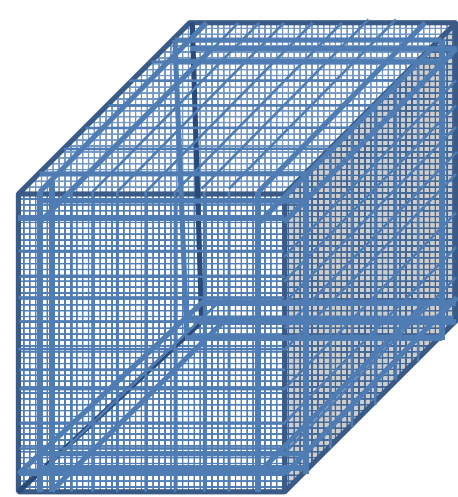

(a)

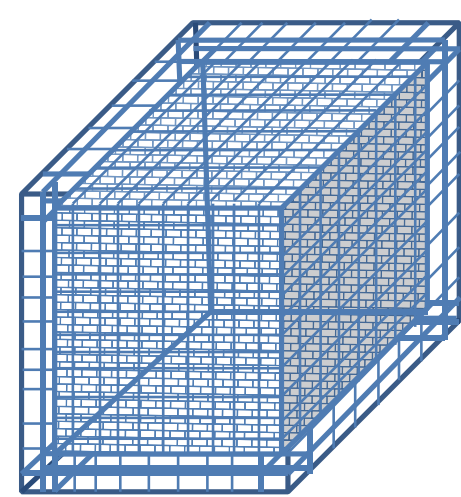

(b)

FIGURE 3: UPML layers around the FDTD mesh with (a) all layers and (b) removed frontal layers. The FDTD mesh is highlighted.

TABLE 1: Characteristics of microstrip inset patch antenna.

\begin{tabular}{lccc}
\hline Component & Width $(\mathrm{mm})$ & Length $(\mathrm{mm})$ & Thickness $(\mathrm{mm})$ \\
\hline Ground plane (copper) & 16.8 & 24.5 & 0.5 \\
Patch (copper) & 8.0 & 10.0 & 0.5 \\
Substrate (duroid 5880) & 16.6 & 28.5 & 3.5 \\
Feed line (copper) & 1.5 & 17.0 & 0.5 \\
\hline
\end{tabular}

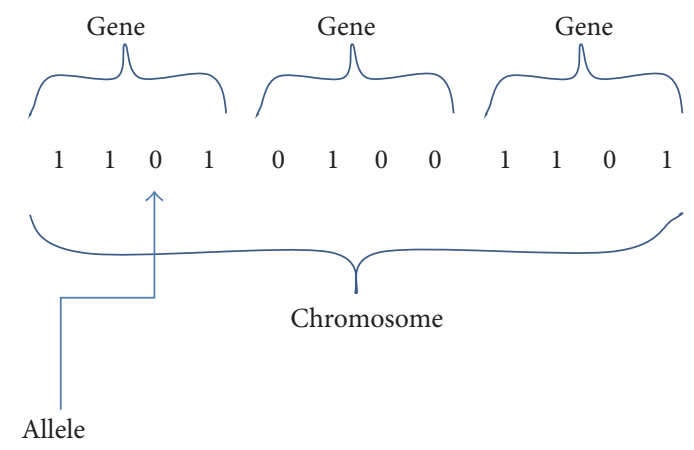

FIGURE 4: Sample of a binary chromosome.

parameters are given in Table 1. The origin of the coordinate system was set at the center of the ground plane, at zero height on the base.

4.2. FDTD Considerations. The FDTD method works in the time domain. Thus, the use of signals with variant spectrum is recommended. There are three approaches to this method: total field/scattered field formulation; additive source; and hard-source. Since the energy is introduced into the spacetime lattice, the hard-source solution was used. This is incorporated into the FDTD code by specifying the electric field value at a specific location by a time function (typically Gaussian or a Gaussian derivative), which takes advantage of the wideband nature of the problem. In this study, Morlet's wavelet function was used. The driving field is given by

$$
E_{z}(t)=E_{0} \exp \left[-\left(2 \pi f_{b}\left(t-t_{0}\right)\right)^{2}\right] \cos \left[2 \pi f_{c}\left(t-t_{0}\right)\right]
$$

with $E_{0}=12 \mathrm{~V} / \mathrm{m}, t_{0}=375 \mathrm{ps}, f_{c}=14.25 \mathrm{GHz}$, and $f_{b}=$ $2000 \mathrm{MHz}$, where $E_{0}$ is the signal amplitude, $t_{0}$ is the time at which the pulse reaches its maximum value, $f_{c}$ is the center frequency, and $t$ represents the propagation time signal with $0 \leq t \leq 8000 \Delta t$, where $\Delta t=0.0342 \mathrm{~ns}$ is the time step. This signal is shown in Figure 7. The spatial steps $\Delta x=0.234 \mathrm{~mm}$, $\Delta y=0.21 \mathrm{~mm}$, and $\Delta z=0.11 \mathrm{~mm}$ were used. A mesh is generated by dividing the computational domain into $72 \times$ $113 \times 39$ tiny cells. The time step is calculated according to Courant criterion (see (2)). 8000 iterations were necessary to address the steady-state, rendering approximately 9 minutes of computer time.

4.3. Validation. The software implemented was verified by comparing the results for a common microstrip inset patch antenna with those obtained from the commercial software package CST-MWS ${ }^{\circledR}$. The magnitudes of $S_{11}$ for both packages are shown in Figure 8 . The nature of the variation of $S_{11}$ obtained with FDTD follows closely that obtained by CSTMWS.

4.4. GA Considerations. For the optimization process, all elements of the initial population refer to a standard rectangular patch antenna with inset (Figure 6). The corresponding resonant patch is divided into a binary matrix of $17 \times$ 17 subpatches (see sample in Figure 9). The subpatches correspond to the intrinsic representation of the problem. This indicates the presence (set as 1) or the absence (set as 0 ) of copper in a candidate solution (chromosome). The idea is that, in the random generation of the initial population, the subpatches to be generated have behavior similar to that of the passive resonating elements. Considering that both the FDTD and GA are time-consuming processes, various 


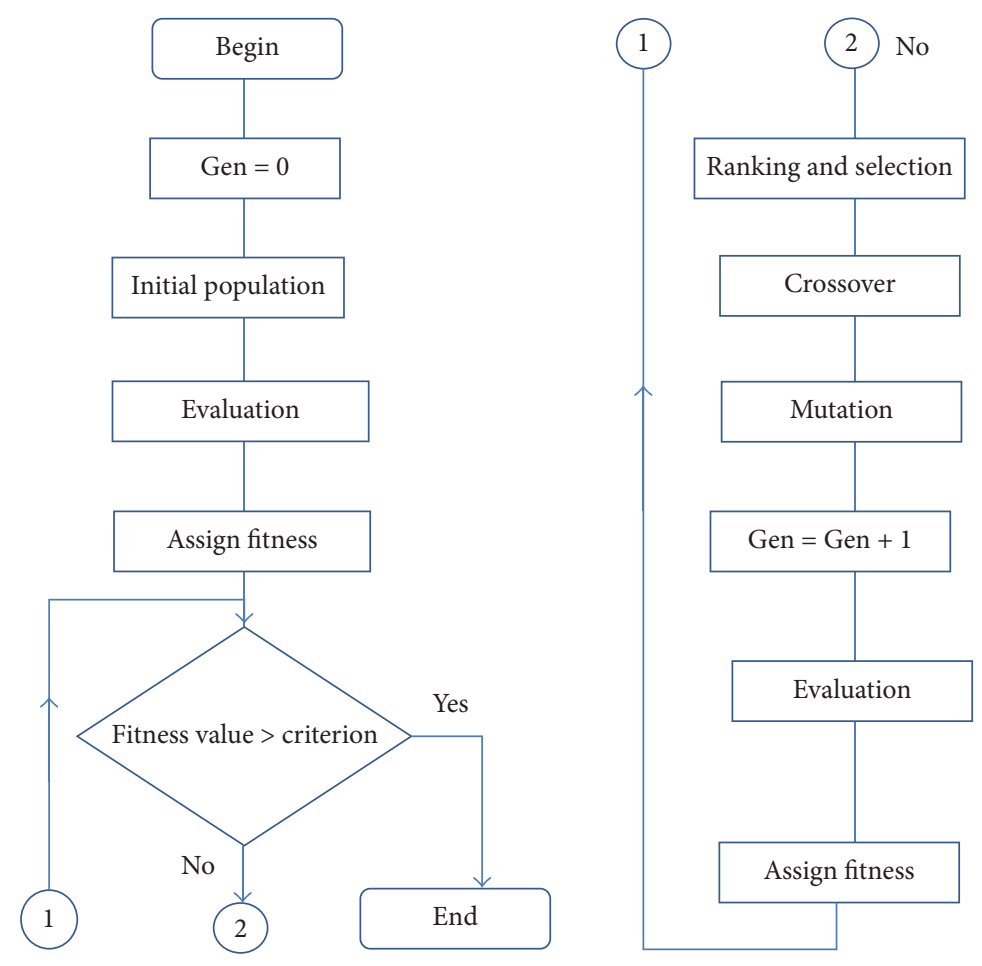

FIGURE 5: Flowchart of the basic genetic algorithm.
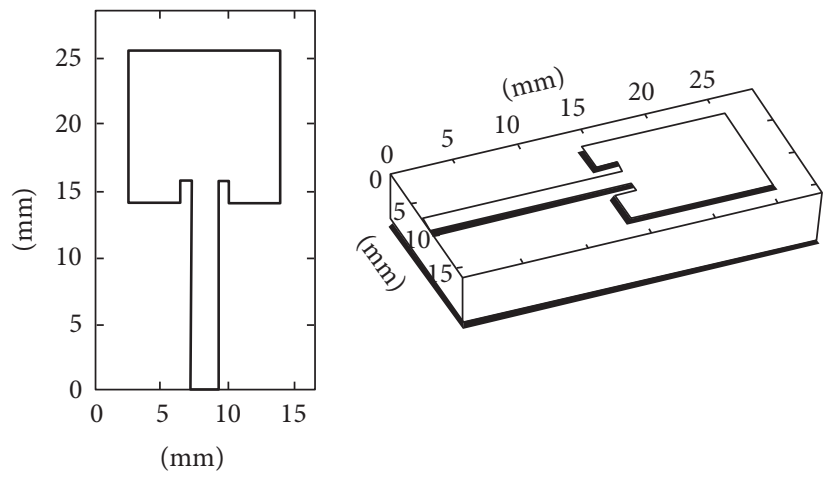

FIGURE 6: Microstrip antenna with inset (unscaled).

chromosome sizes were tested. A digital individual with 289 elements showed a good balance between accuracy and computational burden.

Each chromosome is composed of 289 alleles. Within the program a one-dimensional binary array represents the irregular patch antenna. Each subpatch is an exact multiple of the size of the corresponding dimensional step in order to create a second $17 \times 17$ mesh of subpatches. The latter is stored in a two-dimensional array. The corresponding conversion from vector to matrix and vice versa is done through a subroutine.

The initial size of the population was set at 70 chromosomes (all different). This number should be sufficient to ensure the generation of good parents (i.e., with sufficient diversity to ensure that some chromosomes have the desired
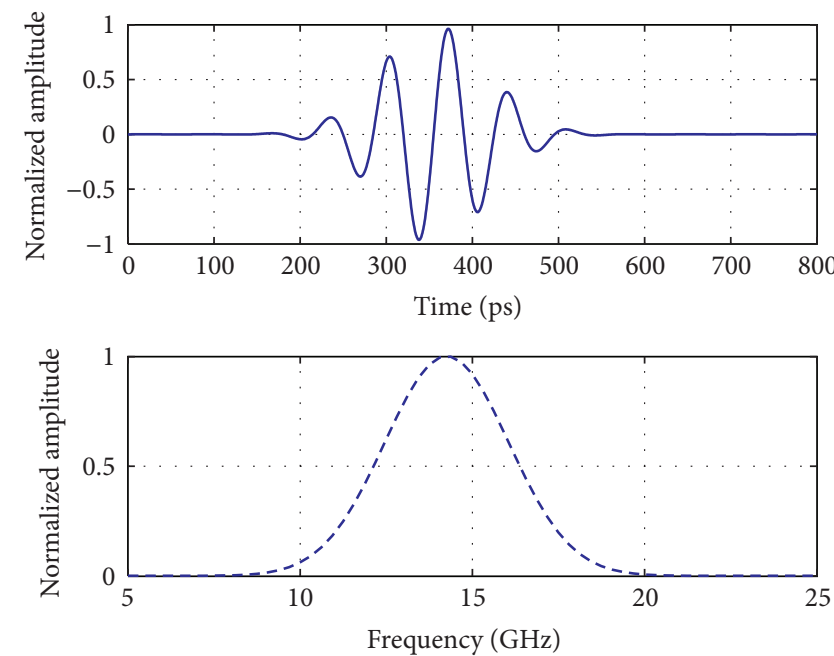

FIgURE 7: Signal excitation in time and frequency domains.

characteristics). For the evaluation of each population, the time consumed was 10.5 hours on a PC with i7 processor, 12 GB of RAM, and Nvidia $\odot$ GeFORCE GT 610 Graphics Accelerator, with 2 GB DDR3/ 64 bits.

Another consideration is related to the fitness function used to evaluate if the individual is a good solution. Each chromosome in a population has a fitness value associated with it and the chromosomes are ranked from best to worst based on this value. This function is considered to be the most important part of the algorithm, because its success 


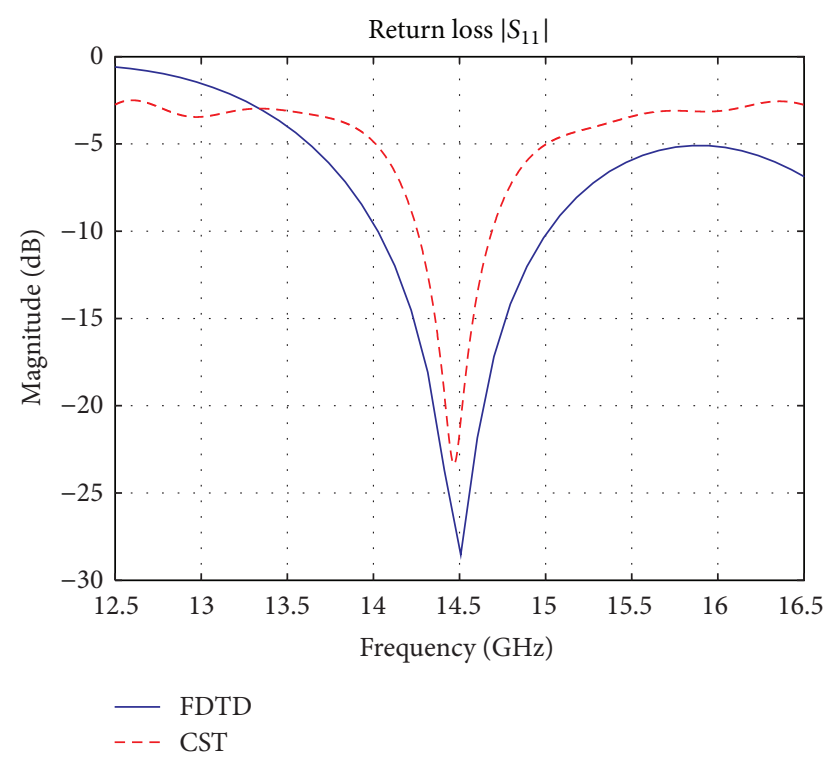

FIGURE 8: Simulated results for the return loss of a rectangular patch antenna with CST and the developed simulator.

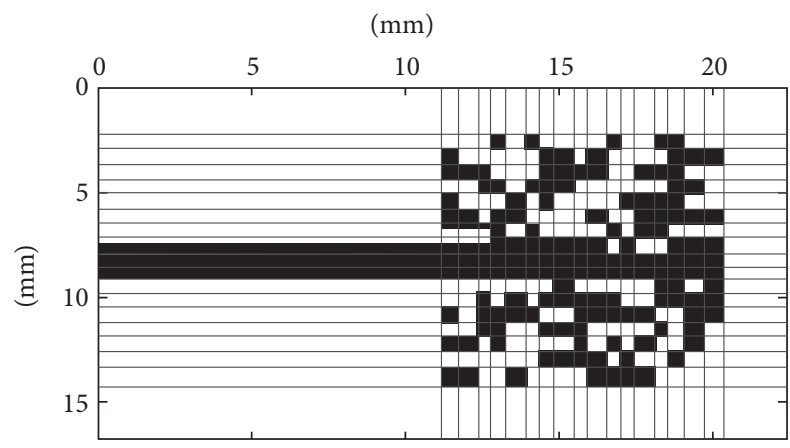

FIGURE 9: Patch antenna configuration in the evolutionary process.

is dependent on how well the fitness function evaluates each solution in relation to the overall objectives of the optimization problem. The fitness function is related to the bandwidth and the return loss (see (5)). The two optimization objectives functions were combined to form a single overall fitness function.

In (5), $\alpha$ is a weighting factor in the range $[0,1]$. This parameter allows us to choose the emphasis or preference of one parameter with respect to another. The fitness function related to bandwidth is set to a minimum bandwidth of $2.0 \mathrm{GHz}$, as in the study described in [30], and the related return loss value is better than $20 \mathrm{~dB}$, as reported in [31].

$$
\text { fit }=\alpha \text { fitBW }+(1-\alpha) \text { fitPR. }
$$

For the application of the basic genetic operators, that is, selection, crossover, and mutation, the specific bit operator roulette wheel, single point crossover, and elitism, were used. In the roulette wheel, each member of the new population is selected in a random way from the elements of the previous population, where the probability of selecting a member from the previous population is proportional to its fitness value. Crossover involves creating two "offspring" chromosomes from the complementary part of two "parent" chromosomes. A random point is chosen, the two parents are then divided, and in the left side the first parent becomes the left side of second parent and vice versa. A crossover probability controls the frequency of the operator, which was fixed at 0.8. In the mutation process, the operator mutation randomly flips some of the bits in a chromosome. Mutation probability was fixed at 0.05 , changing the offspring properties. Crossover and mutation are important operators that are responsible for improving the diversity.

Elitism is the process of retaining or inserting the best individual into the next generation from the current generation. This is performed to avoid the loss of the best individual in any population during the crossover and mutation operations. Thus, using these operators, good candidates are retained while others are discarded in such a way that the population size remains constant.

\section{Results}

Figure 10 shows snapshots of the iterative evolutionary process, together with their respective $S_{11}$ graphs $(\mathrm{dB})$. Wideband and high return loss were the optimization criteria. Figures 10(a) and 10(b) show two instants in the evolutionary process in which the corresponding chromosomes have distinct characteristics. Figure 10(a) shows a return loss of the order of $26 \mathrm{~dB}$ and a poor bandwidth of $1 \mathrm{GHz}$, while Figure 10(b) shows a poor return loss but frequency response with a wideband behavior. These two chromosomes are the arguments in the crossover process that will generate the next individuals. The antennas in Figures 10(c) and 10(d) were the best solutions. In Figure 10(c) the peak value for the return loss is $27.04 \mathrm{~dB}$, with a $10 \mathrm{~dB}$ bandwidth of $6 \mathrm{GHz}$. In Figure $10(\mathrm{~d})$, the values for the return loss are around $35 \mathrm{~dB}$, with a $10 \mathrm{~dB}$ bandwidth of $3.7 \mathrm{GHz}$, and better return loss is observed in the middle frequencies.

For the antenna of Figure 10(d), the corresponding radiation patterns are presented in Figure 11, at $13 \mathrm{GHz}$, $14.25 \mathrm{GHz}$, and $15 \mathrm{GHz}$, corresponding to the columns of the figure. This antenna presents $15 \mathrm{~dB}$ minimum return loss at ATG frequency range. The $X Y$ plane (azimuth) responses are shown in Figures 11(a), 11(b), and 11(c); the $X Z$ plane responses are shown in Figures 11(d), 11(e), and 11(f); and the $Y Z$ plane responses are shown in Figures 11(g), 11(h), and 11(i).

\section{Conclusions}

In this study, a compact irregularly shaped microstrip patch antenna for use in ATG devices was designed. Excellent return loss and bandwidth values were obtained, with $10 \mathrm{~dB}$ bandwidths up to $6 \mathrm{GHz}$, and peak return loss of around $27 \mathrm{~dB}$. The best antenna showed $15 \mathrm{~dB}$ minimum return loss over the full ATG frequency band. The finite-difference timedomain method (FDTD) was used in conjunction with a genetic algorithm. The proposed methodology can be applied 

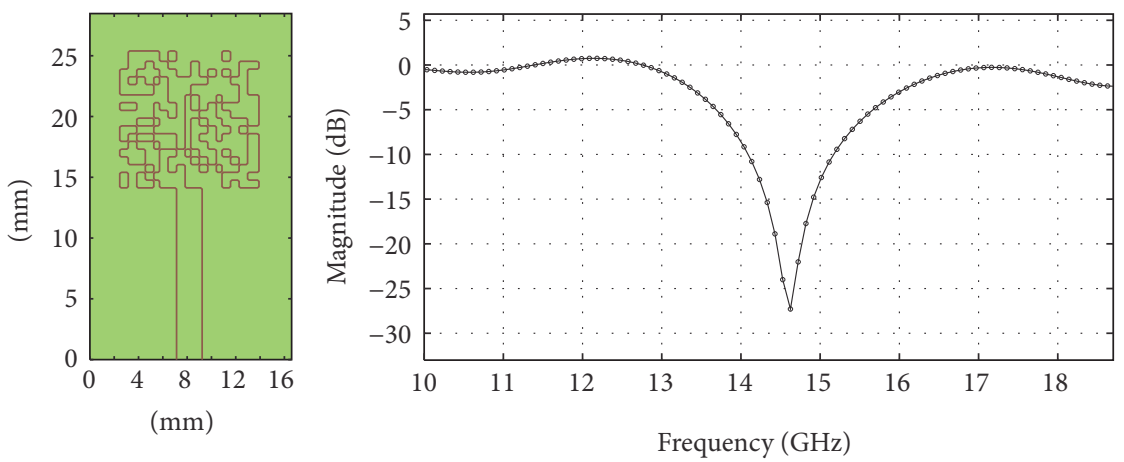

(a)
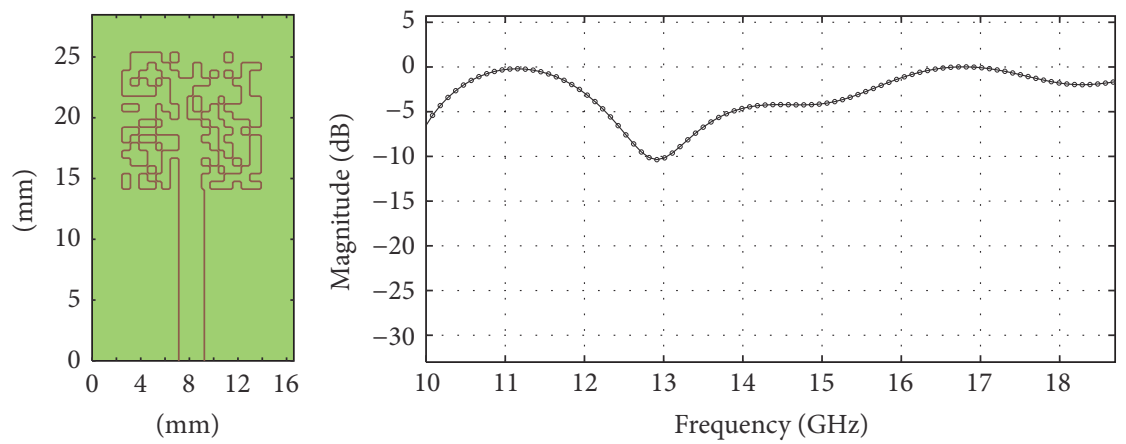

(b)
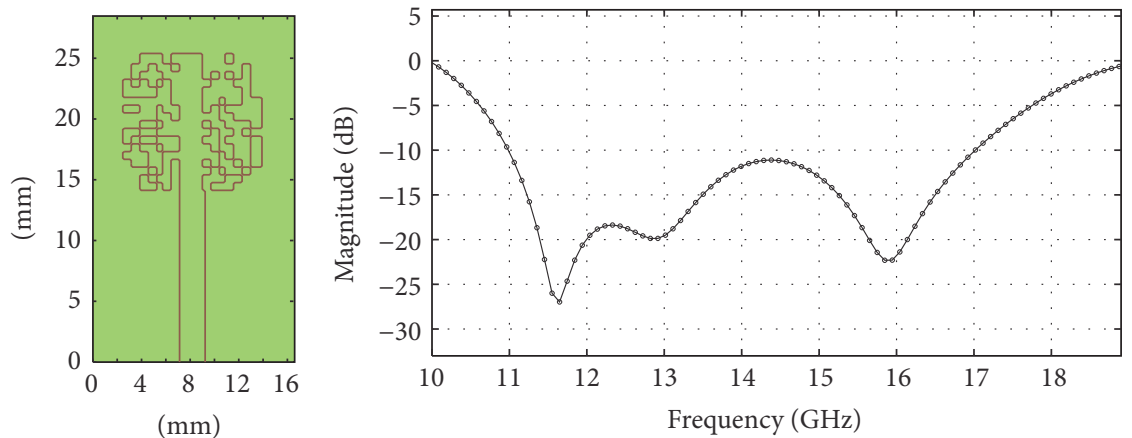

(c)
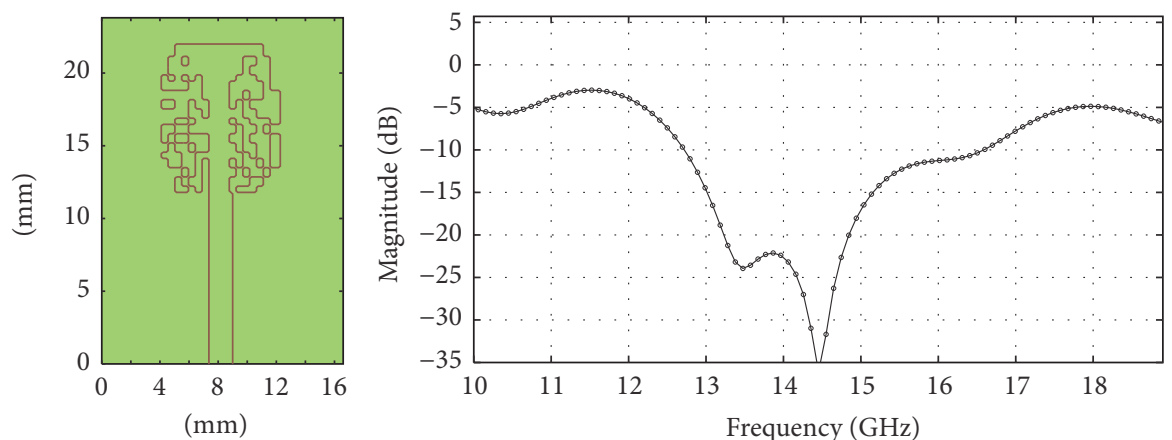

(d)

FIGURE 10: Some snapshots from the evolutionary optimization process. 


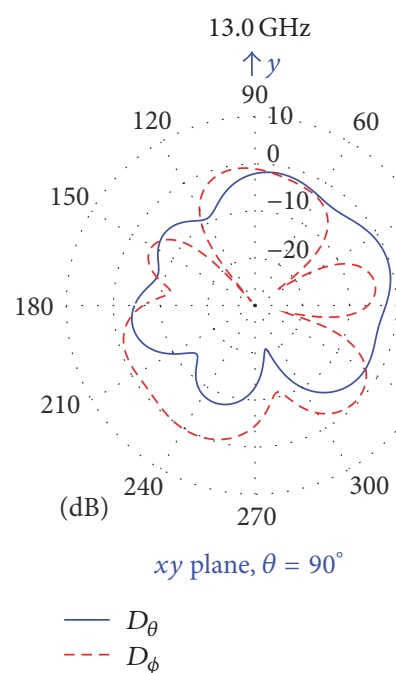

(a)

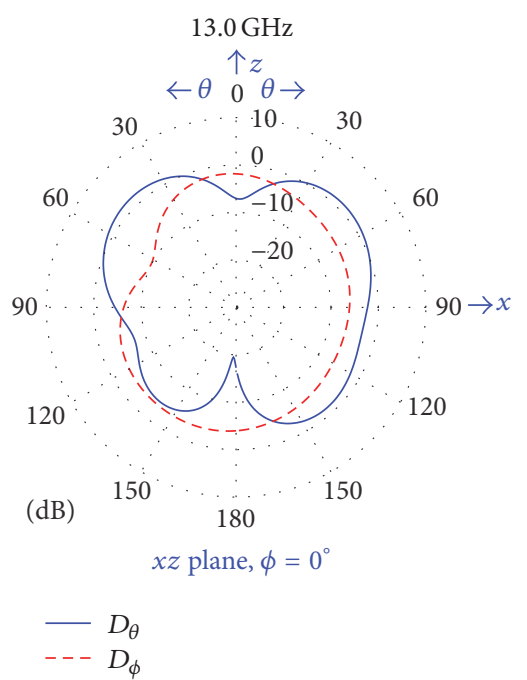

(d)

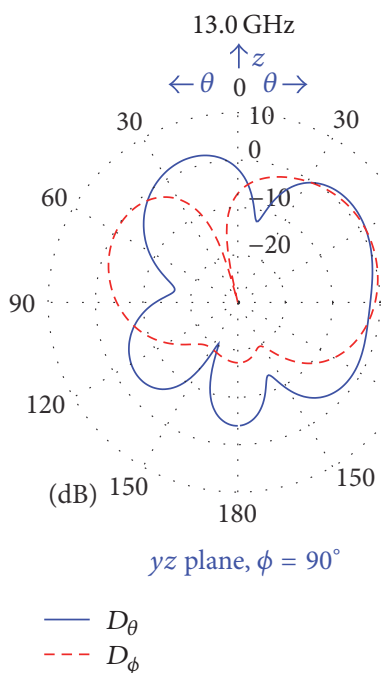

(g)
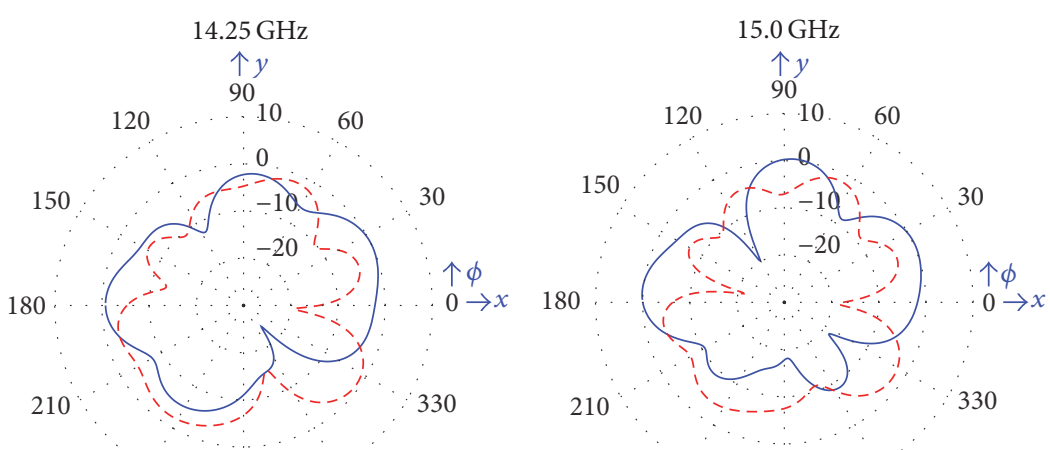

(dB) $240 \quad \ldots \ldots+300$

$x y$ plane, $\theta=90^{\circ}$

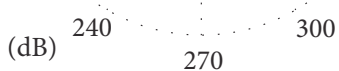

$x y$ plane, $\theta=90^{\circ}$

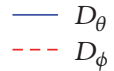

(b)

(c)

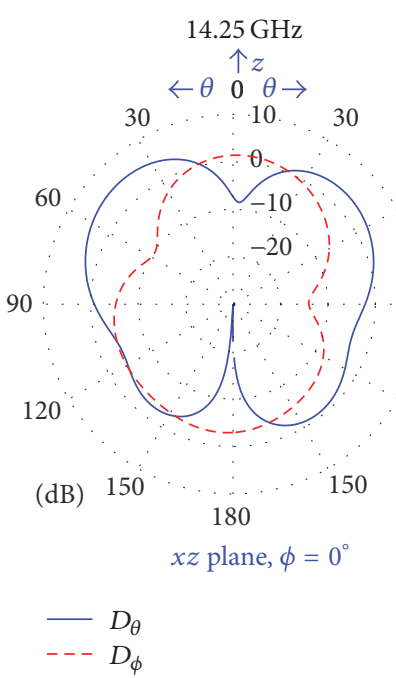

(e)

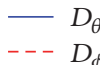

$D_{\theta}$
$D_{\phi}$
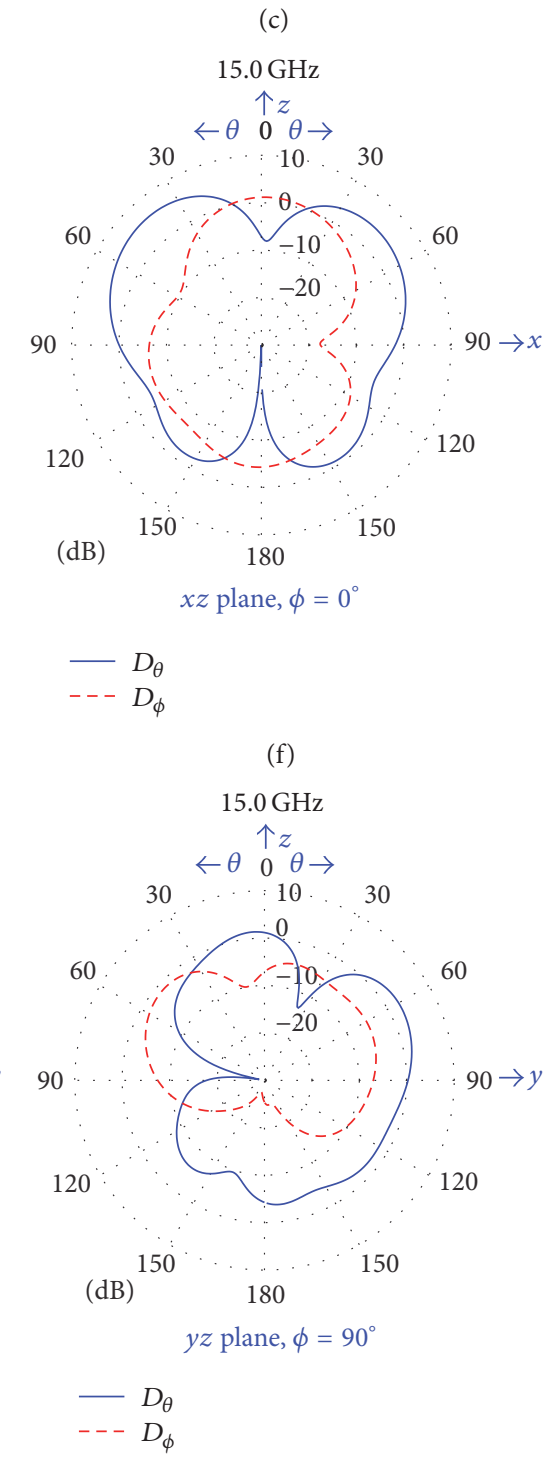

$$
\begin{aligned}
& \text { (dB) }{ }^{150} \quad \begin{array}{lll}
180 & 150
\end{array} \\
& y z \text { plane, } \phi=90^{\circ} \\
& \begin{array}{c}
-D_{\theta} \\
---D_{\phi}
\end{array}
\end{aligned}
$$
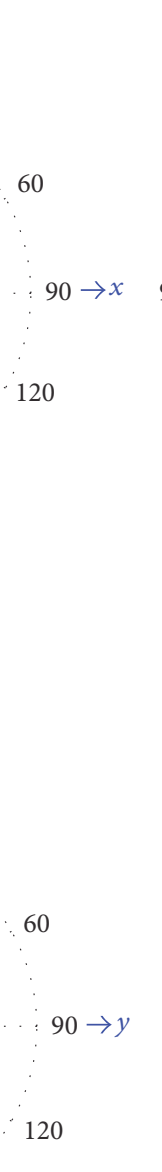

(h)

(i)

FiguRE 11: Radiation patterns of the antenna of Figure 10(d) for 13, 14.25, and $15 \mathrm{GHz}$. 
to the design of other microstrip antennas. This is currently under investigation.

\section{Conflicts of Interest}

The authors declare that there are no conflicts of interest regarding the publication of this paper.

\section{Acknowledgments}

This work was supported by CAPES PNPD Scholarship 20131064.

\section{References}

[1] S. R. Saunders, Antennas and Propagation for Wireless Communication Systems, John Wiley \& Sons, 2003.

[2] G. A. Deschamps and W. Sichak, "Microstrip microwave antennas," in Proceedings of the 3rd Symposium on the USAF Antennas Research and Development Program, pp. 18-22, October 1953.

[3] I. J. Bahl and P. Bhartia, Microstrip Antennas, Artech House, Dedham, Mass, USA, 1980.

[4] J. R. James and P. S. Hall, Handbook of Microstrip Antennas, Peter Peregrinus, London, UK, 1989.

[5] D. M. Pozar and D. H. Schaubert, Microstrip Antennas, IEEE Press, New York, NY, USA, 1995.

[6] J. F. Zurcher and F. E. Gardiol, Broadband Patch Antennas, Artech House, Boston, Mass, USA, 1995.

[7] K. F. Lee and W. Chen, Advances in Microstrip and Printed Antennas, John Wiley \& Sons, New York, NY, USA, 1997.

[8] R. Garg, P. Bhartia, I. Bahl, and A. Ittipiboon, Microstrip Antenna Design Handbook, Artech House, Boston, Mass, USA, 2001.

[9] R. B. Waterhouse, Microstrip Patch Antennas: A Designer's Guide, Springer, Berlin, Germany, 2003.

[10] R. Bancroft, Microstrip and Printed Antenna Design, Noble Publishing, 2004.

[11] K. Wong, Compact and Broadband Microstrip Antennas, John Wiley \& Sons, New York, NY, USA, 2002.

[12] G. Kumar and K. P. Ray, Broadband Microstrip Antennas, Artech House, Boston, Mass, USA, 2002.

[13] Z. N. Chen and M. Y. W. Chia, Broadband Planar Antennas: Design and Applications, John Wiley \& Sons, New York, NY, USA, 2006.

[14] P. S. Hall and Y. Hao, Antennas and Propagation for Body-Centric Wireless Communications, Artech House, Boston, Mass, USA, 2006.

[15] D. Guha and Y. M. M. Antar, Microstrip and Printed Antennas New Trends, Techniques and Applications, John Wiley \& Sons, 2011.

[16] S. Narayan, B. Sangeetha, and R. M. Jha, Frequency Selective Surfaces Based High Performance Microstrip Antenna, Springer, Singapore, 2016.

[17] J. Michael Johnson and Y. Rahmat-Samii, "Genetic algorithms and method of moments (GA/MOM) for the design of integrated antennas," IEEE Transactions on Antennas and Propagation, vol. 47, no. 10, pp. 1606-1614, 1999.

[18] H. Choo, A. Hutani, L. C. Trintinalia, and H. Ling, "Shape optimization of broadband microstrip antennas using genetic algorithm," Electronics Letters, vol. 36, no. 25, pp. 2057-2058, 2000 .

[19] C. A. Balanis, Modern Antenna Handbook, John Wiley \& Sons, 2011.

[20] L. A. Griffiths, C. Furse, and Y. C. Chung, "Broadband and multiband antenna design using the genetic algorithm to create amorphous shapes using ellipses," IEEE Transactions on Antennas and Propagation, vol. 54, no. 10, pp. 2776-2782, 2006.

[21] J. Geng, R. Jin, X. Liang, H. Wu, Y. Sheng, and B. Zhou, "The study on the antenna optimization," in Proceedings of the Progress in Electromagnetics Research Symposium (PIERS '10), pp. 22-26, Xi'an, China, February 2010.

[22] A. Modiri and K. Kiasaleh, "Efficient design of microstrip antennas for SDR applications using modified PSO algorithm," IEEE Transactions on Magnetics, vol. 47, no. 5, pp. 1278-1281, 2011.

[23] D. Minoli, Innovations in Satellite Communications and Satellite Technology, John Wiley \& Sons, Hoboken, NJ, USA, 2015.

[24] M. Kowalke, "New Air-to-Ground 4G LTE System Could Put Ultra-Broadband in the Skies," Next Generation Communications, July 2015, http://next-generation-communications.tmcnet.com/topics/industries/articles/407374-new-air-toground-4g-lte-system-could.htm.

[25] L. Afonso, N. Souto, P. Sebastiao, M. Ribeiro, T. Tavares, and R. Marinheiro, "Cellular for the skies: exploiting mobile network infrastructure for low altitude air-to-ground communications," IEEE Aerospace and Electronic Systems Magazine, vol. 31, no. 8, pp. 4-11, 2016.

[26] L. A. R. Ramirez, Aplicação do Método das Diferenças Finitas no Domínio do Tempo na Análise de Cobertura em Ambientes Interiores [Ph.D. thesis], Pontifical Catholic University of Rio de Janeiro, 2008 (Portuguese).

[27] S. D. Gedney, "An anisotropic perfectly matched layerabsorbing medium for the truncation of FDTD lattices," IEEE Transactions on Antennas and Propagation, vol. 44, no. 12, pp. 1630-1639, 1996.

[28] W. Pedrycz, Handbook of Computational Intelligence, Springer, 2015.

[29] C. A. Balanis, Antenna Theory Analysis and Design, John Wiley \& Sons, 1997.

[30] J. Kim, T. Yoon, J. Kim, and J. Choi, "Design of an ultra wide-band printed monopole antenna using FDTD and genetic algorithm," IEEE Microwave and Wireless Components Letters, vol. 15, no. 6, pp. 395-397, 2005.

[31] S. Sun, L. V. Yinghua, and J. Zhang, "The application of genetic algorithm optimization in broadband microstrip antenna design," in Proceedings of the IEEE 5th Asia-Pacific Conference on Environmental Electromagnetics, pp. 192-195, September 2010. 


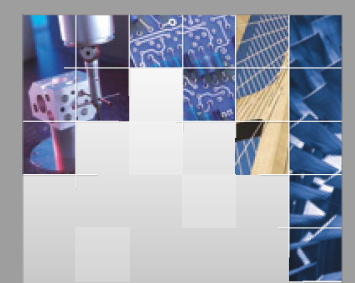

\section{Enfincering}
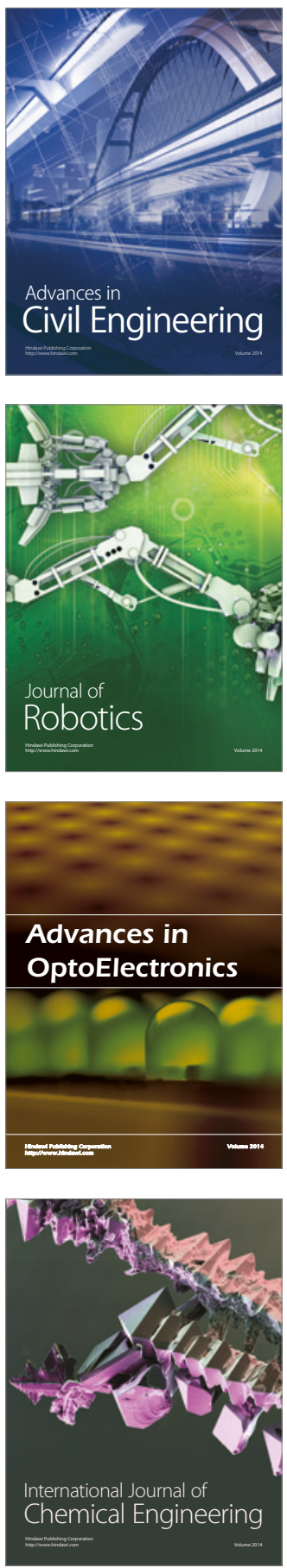

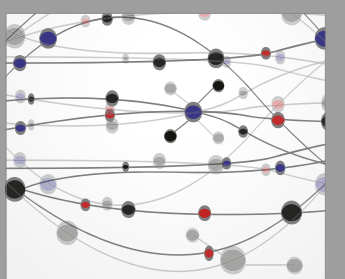

The Scientific World Journal

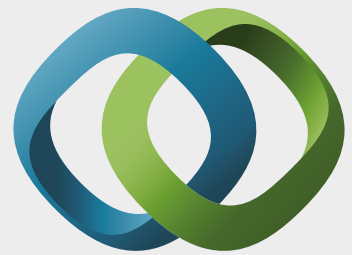

\section{Hindawi}

Submit your manuscripts at

https://www.hindawi.com
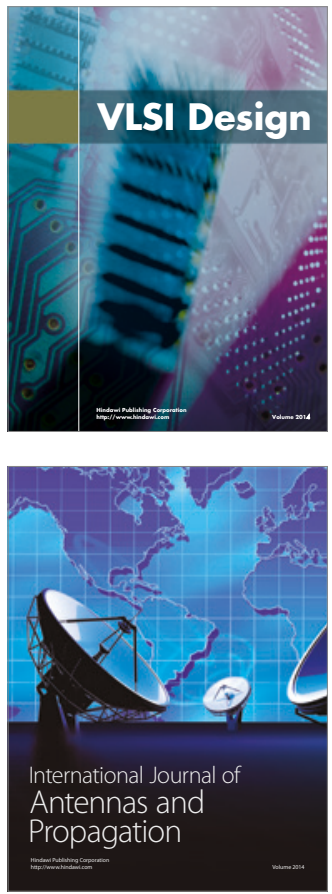

\section{Rotating}

Machinery
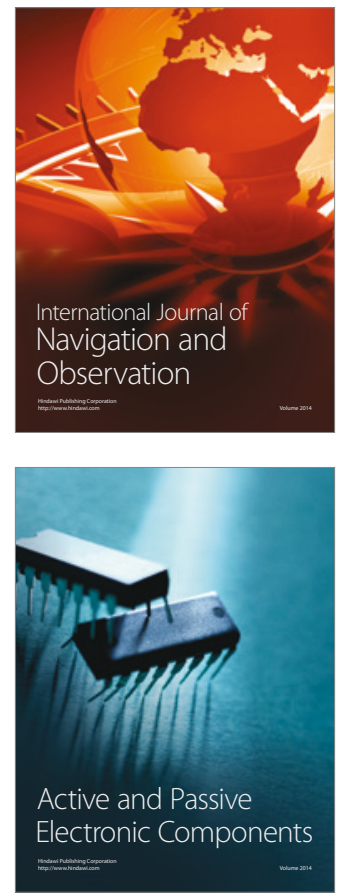
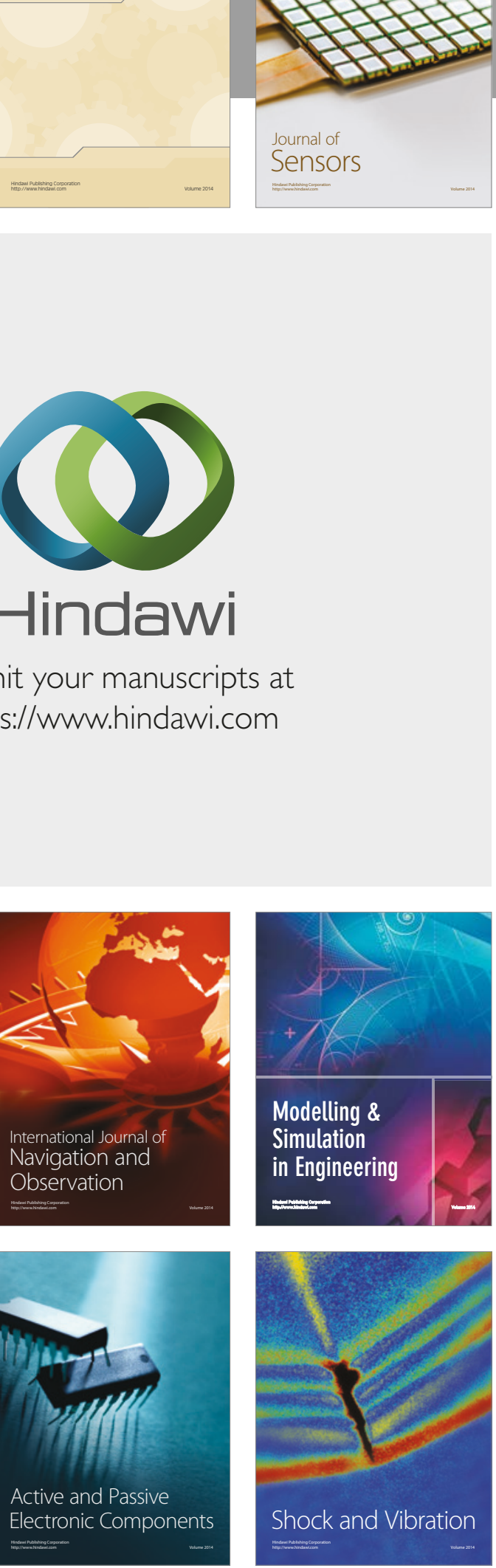
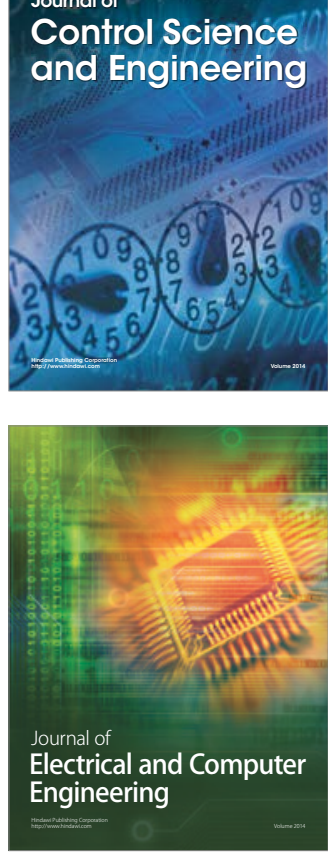

Distributed

Journal of

Control Science

and Engineering
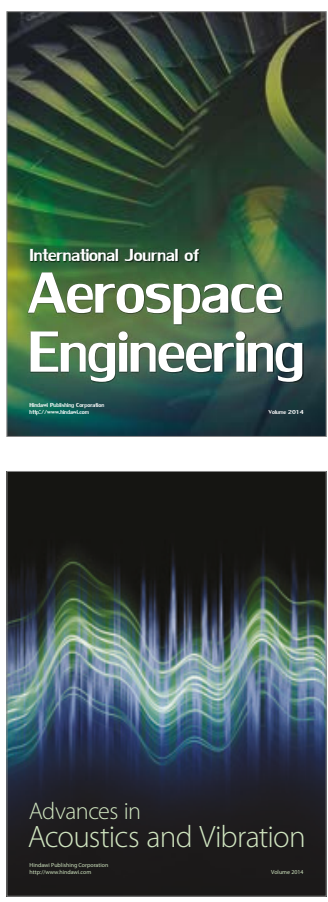

Sensor Networks 